\title{
Percutaneous versus Transcutaneous Electrical Stimulation of the Posterior Tibial Nerve in Idiopathic Overactive Bladder Syndrome with Urinary Incontinence in Adults: A Systematic Review
}

\author{
Aida Agost-González ${ }^{1}$, Isabel Escobio-Prieto ${ }^{1, * \mathbb{D}}$, Azahara M. Pareja-Leal ${ }^{2}$, María Jesús Casuso-Holgado ${ }^{1} \mathbb{D}$, \\ María Blanco-Diaz ${ }^{3}$ and Manuel Albornoz-Cabello ${ }^{1}$ (D) \\ 1 Departamento de Fisioterapia, Facultad de Enfermería, Fisioterapia y Podología, Universidad de Sevilla, \\ 41009 Sevilla, Spain; aidaagost97@gmail.com (A.A.-G.); mcasuso@us.es (M.J.C.-H.); \\ malbornoz@us.es (M.A.-C.) \\ 2 Servicio Madrileño de Salud, D.A. Norte, 28035 Madrid, Spain; azaharapl@gmail.com \\ 3 Departamento de Fisioterapia, Facultad de Medicina y Ciencias de la Salud, Universidad de Oviedo, \\ 33006 Oviedo, Spain; blancomaria@uniovi.es \\ check for \\ * Correspondence: iescobio@us.es
}

updates

Citation: Agost-González, A.; Escobio-Prieto, I.; Pareja-Leal, A.M.; Casuso-Holgado, M.J.;

Blanco-Diaz, M.; Albornoz-Cabello, M. Percutaneous versus Transcutaneous Electrical Stimulation of the Posterior Tibial Nerve in Idiopathic Overactive Bladder Syndrome with Urinary Incontinence in Adults: A Systematic Review. Healthcare 2021, 9, 879. https:// doi.org/10.3390/healthcare9070879

Academic Editors: Grigorios Nasios, Lambros Messinis, Efthimios Dardiotis and Markos Sgantzos

Received: 6 June 2021

Accepted: 11 July 2021

Published: 13 July 2021

Publisher's Note: MDPI stays neutral with regard to jurisdictional claims in published maps and institutional affiliations.

Copyright: (c) 2021 by the authors. Licensee MDPI, Basel, Switzerland. This article is an open access article distributed under the terms and conditions of the Creative Commons Attribution (CC BY) license (https:/ / creativecommons.org/licenses/by/ $4.0 /)$.
Abstract: Background: Percutaneous electrical stimulation and transcutaneous electrical stimulation (PTNS and TTNS) of the posterior tibial nerve are internationally recognized treatment methods that offer advantages in terms of treating patients with overactive bladder (OAB) who present with urinary incontinence (UI). This article aims to analyze the scientific evidence for the treatment of OAB with UI in adults using PTNS versus TTNS procedures in the posterior tibial nerve. Methods: A systematic review was conducted, between February and May 2021 in the Web of Science and Scopus databases, in accordance with the PRISMA recommendations. Results: The research identified 259 studies, 130 of which were selected and analyzed, with only 19 used according to the inclusion requirements established. The greatest effectiveness, in reducing UI and in other parameters of daily voiding and quality of life, was obtained by combining both techniques with other treatments, pharmacological treatments, or exercise. Conclusions: TTNS has advantages over PTNS as it is more comfortable for the patient even though there is equality of both therapies in the outcome variables. More research studies are necessary in order to obtain clear scientific evidence.

Keywords: percutaneous electric nerve stimulation; transcutaneous electric nerve stimulation; adult; urinary bladder; overactive; urinary incontinence; tibial nerve

\section{Introduction}

The International Classification of Diseases (ICD-11) defines overactive bladder syndrome (OAB), with code GC50.0, as a urological condition characterized by voiding urgency, polyuria, and nocturia that may or may not be accompanied by urinary incontinence (UI) [1]. OAB presents a worldwide prevalence [2] of $16 \%$ to $23 \%$, rising to $15 \%$ in those over the age of 40 years [3] and to $30-40 \%$ in those over 75 years [4], although it can be found in people of all ages. Its prevalence in Europe [5] is around 12\%.

J.C. Angulo, in an article [6] published in 2016, revealed a $19.46 \%$ prevalence of OAB in the Spanish population, with at least one episode of urge UI (UUI) a day in $48.74 \%$ of cases [6]. This prevalence is greater in females than in males. Studies conducted [7] in the populations of Europe, the United States, Asia, and Africa reveal a prevalence of UUI of $1.5 \%$ to $14.3 \%$ in men aged between 18 and 20 years, whereas in women, it ranges from $1.6 \%$ to $22.8 \%$. The same is true for those aged over 30 years, where the prevalence in men is from $1.7 \%$ to $13.3 \%$, as opposed to $7 \%$ to $30.3 \%$ in women [7]. 
Among subjects with $\mathrm{OAB}$ on the global scale, UUI has been observed to be the most unpleasant symptom of this condition [4]. Further, people with OAB usually adopt certain coping strategies that involve a decrease in their quality of life and socialization, such as limiting liquid intake, avoiding traveling, and attempting to have direct access to toilets [3].

$\mathrm{UI}$ is the involuntary leakage of urine and lack of ability to control urination, accompanied by spontaneous contractions of the detrusor muscle. There are various subtypes of UI: urgency UI (UII), the sudden desire or need to urinate; stress UI (SUI), caused by efforts, physical exercise, sneezing, or coughing; mixed UI (MUI), combined with urgency and efforts [1] (code MF50.2 in ICD-11). To be able to determine the best treatment option in each patient, a personalized assessment is necessary, including the evaluation of different aspects of health, motivation, and availability or access to specific treatments [2].

Profitability is a fundamental aspect when it comes to reviewing treatment options in this type of condition, which entail great social and financial costs. Previous studies [7] showed a value of EUR 7 billion in subjects with OAB over 18 years old in Canada and European countries, including Spain [7].

There are several alternatives for $\mathrm{OAB}$ and UI treatment: behavioral treatments, considered first-line treatments; pharmacological or second-line treatments such as anticholinergic or antimuscarinic and b-adrenergic drugs, and, by way of a third line of treatment, injections of OnabotulinumtoxinA and therapies with electrical stimulation, including, among others, percutaneous and transcutaneous electrical stimulation (PTNS and TTNS, respectively), which are the object of this study [8].

With regard to treatment by electrical stimulation of the posterior tibial nerve (PTN), this involves retrograde stimulation of the nerve fibers of the sacral plexus, which innervates the bladder and detrusor muscle $[2-5,8]$. Electrical stimulation can be applied through insertion of a needle in the PTN—that is, PTNS is carried out in the said nerve-or through surface electrodes, with TTNS [9], with beneficial and safe effects in the short term in women with $\mathrm{OAB}$, and no relevant adverse effects [10], according to the review by Sousa-Fraguas et al., 2020.

These techniques may represent an advantage in treatment of subjects with $\mathrm{OAB}$ who present UI, enabling these difficulties to be solved, as they can be compared favorably to treatment using antimuscarinic drugs, due to them being less costly [11].

In this respect, the present study aimed to summarize the knowledge available and conduct a critical analysis of the evidence from randomized controlled clinical trials, observational studies, systematic reviews, and meta-analyses on the effectiveness of PTNS and TTNS in the treatment of adults with OAB who present UI.

\section{Materials and Methods}

This systematic review followed the Preferred Reporting Items for Systematic Reviews and Meta-Analyses (PRISMA) guidelines [12]. The protocol was registered in the International Prospective Register of Systematic Reviews (PROSPERO/NHS)—number: 184809.

\subsection{Selection Criteria}

Three researchers independently reviewed the articles found. In order to formulate the objective and the question of the review, the PICOS strategy was used [13] (P-population or patients; I-intervention; $\mathrm{C}$-comparison; $\mathrm{O}$-outcomes; $\mathrm{S}$ - study design), in which $\mathrm{P}=$ (adults with $\mathrm{OAB}$ syndrome (OABS) and presence of $\mathrm{UI}) ; \mathrm{I}=(\mathrm{PTNS}$ and TTNS); $\mathrm{C}=$ (control group that received no intervention or received standard/usual care); $\mathrm{O}=$ (randomized clinical trials (RCTs), descriptive, observational studies, systematic reviews, and meta-analyses), and $S=$ (randomized controlled clinical trials, descriptive observational studies, systematic reviews, and meta-analyses). This strategy enabled the establishment of critical reasoning on the issue [13] and the formulation of the following question: "What is the existing scientific evidence on the treatment of adults diagnosed as having OABS with UI through procedures of PTNS versus TTNS?". 


\subsection{Data Sources}

The bibliographic search was performed between the months of February and May 2021. The search terms used were percutaneous electric nerve stimulation; transcutaneous electric nerve stimulation; adult; urinary bladder, overactive; urinary incontinence; tibial nerve. Two multidisciplinary databases, Scopus and Web of Science (WOS), were used in the search. The search strategy followed is presented in Table 1.

Table 1. Search strategy in WOS and Scopus databases.

\begin{tabular}{|c|c|}
\hline Databases & Search Strategy \\
\hline $\begin{array}{c}\text { WOS } \\
\text { (February-March 2021) } \\
(\text { years 2015/2020) }\end{array}$ & $\begin{array}{l}\text { I. ("Transcutaneous Electric Nerve Stimulation" OR “Therapy, Percutaneous Neuromodulation" OR } \\
\text { "Electrical Neuromodulation, Percutaneous") AND adult AND ("Urinary Incontinence" OR “Urinary } \\
\text { Bladder, Overactive") AND ("tibial nerve" OR “Posterior tibial nerve"). } \\
\text { II. ("Transcutaneous electrical nerve stimulation" OR “Transcutaneous tibial nerve stimulation" OR } \\
\text { "transcutaneous stimulation tibial nerve") AND adult AND ("overactive bladder" OR "detrusor } \\
\text { activity" OR "urinary incontinence"). } \\
\text { III. ("percutaneous tibial nerve stimulation" OR “PTNS") AND adult AND ("overactive bladder" OR } \\
\text { "detrusor activity" OR "urinary incontinence"). }\end{array}$ \\
\hline $\begin{array}{c}\text { Scopus } \\
\text { (February-March 2021) } \\
(\text { years 2015/2020) }\end{array}$ & $\begin{array}{l}\text { I. ("Transcutaneous Electric Nerve Stimulation" OR “Therapy, Percutaneous Neuromodulation" OR } \\
\text { "Electrical Neuromodulation, Percutaneous") AND adult AND (“Urinary Incontinence" OR “Urinary } \\
\text { Bladder, Overactive") AND ("tibial nerve" OR "Posterior tibial nerve"). } \\
\text { II. ("Transcutaneous electrical nerve stimulation" OR “Transcutaneous tibial nerve stimulation" OR } \\
\text { "transcutaneous stimulation tibial nerve") AND adult AND ("overactive bladder" OR "detrusor } \\
\text { activity" OR "urinary incontinence"). } \\
\text { III. ("percutaneous tibial nerve stimulation" OR "PTNS") AND adult AND ("overactive bladder" OR } \\
\text { "detrusor activity" OR "urinary incontinence"). }\end{array}$ \\
\hline
\end{tabular}

\subsection{Data Collection and Analysis}

By way of exclusion criteria: all articles not published in English or Spanish; studies conducted in patients with neurological diseases or UI exclusively of neurogenic origin; carried out in children, animals, or patients with an associated underlying pathology; addressing fecal incontinence; in which treatment was not carried out with PTNS or TTNS of the PTN, or not aimed at treatment of OAB with UI; narrative or nonsystematic reviews; all documents not aligned with the research problem. The bibliographic research focused on all articles published from 2015 to 2020.

In order to obtain reliable, valid results, without them being influenced by bias, the Physiotherapy Evidence Database scale (PEDro) [14] was used to assess the methodological quality of the experimental studies, based on the Delphi list [15]. In the same way, the STROBE declaration [16] was applied for the evaluation of observational-type studies, and the PRISMA declaration [14] for reviews that followed its criteria in their execution. Articles that did not exceed the score of five in the PEDro scale [14] or with a score of less than 11 points in the STROBE declaration [16] were excluded, finally obtaining the articles chosen for the review.

\section{Results}

\subsection{Literature Search}

Figure 1 shows the PRISMA flow chart of this systematic review.

The initial search in the databases gathered a total of 259 articles, 98 from Web of Science (WOS) and 161 from Scopus.

The initial screening phase produced 130 articles after removing duplicates $(n=129)$.

Based on the titles and abstracts of the articles, a total of 56 articles were removed. Then, considering the remaining 74 eligible studies, many were excluded after full-text reading $(n=47)$, or because it was not possible to access the full text $(n=3)$, or for not passing the methodological quality scale $(n=5)$. 


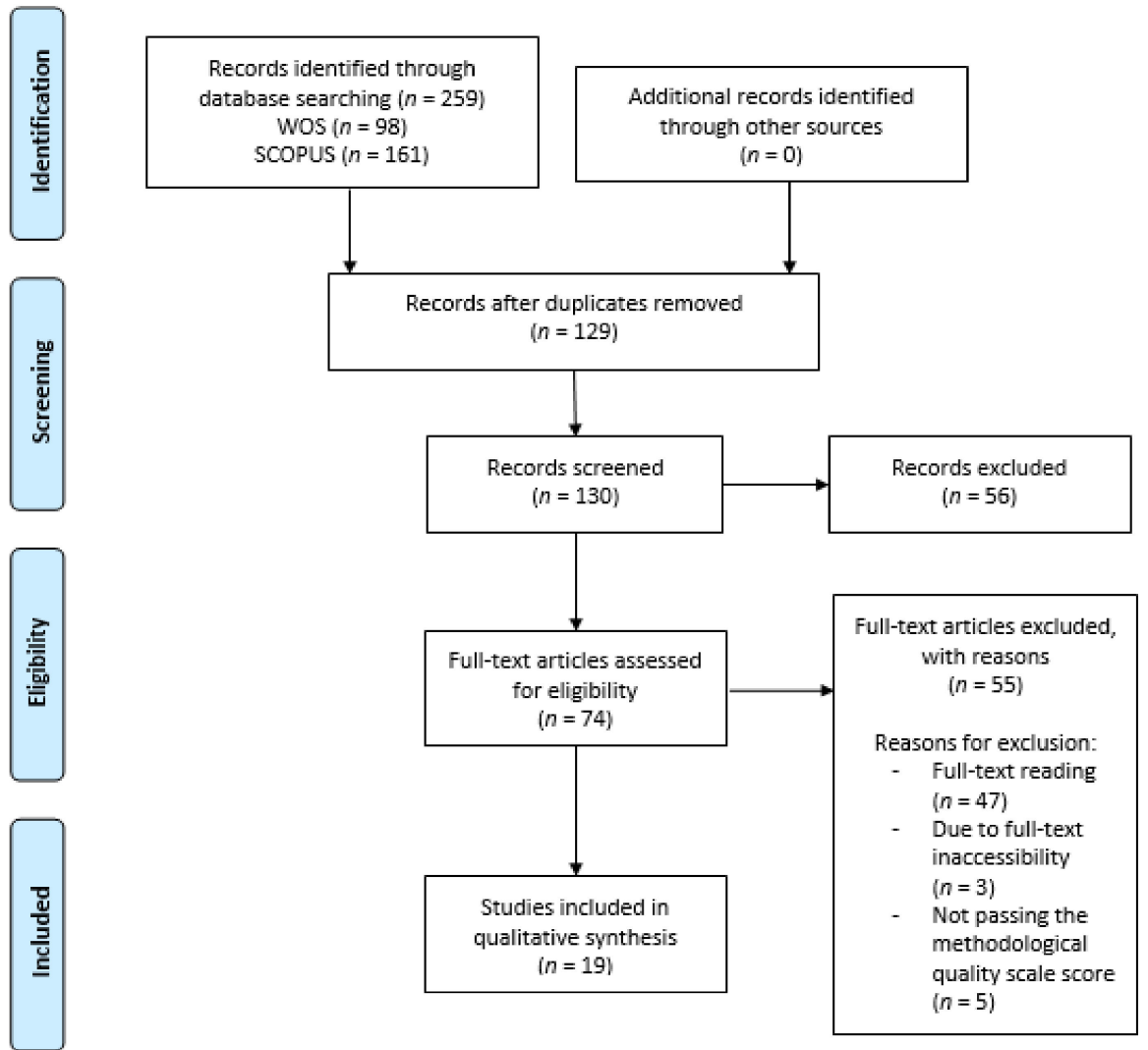

Figure 1. Flow chart of this systematic review.

Finally, 19 studies [3-5,17-32] were included. Of these, nine were experimental [3,17-24], including eight RTCs [3,17-22,24] (Table 2); four were observational studies [5,25-27] (Table 3), and six were either systematic reviews [4,29,31] or a meta-analysis [28], while two encompassed both types of studies [30,32] (Table 4). 
Table 2. Characteristics of experimental studies included in the systematic review. Sevilla, ES. 2021.

\begin{tabular}{|c|c|c|c|c|c|c|}
\hline Author/s & Study Design & Study Population & Sample Size & Intervention & Follow-Up & Randomization \\
\hline $\begin{array}{c}\text { Ramírez-García I., et al., } \\
2019 \text { [17] }\end{array}$ & $\mathrm{RCT}$ & $\mathrm{OAB}$ and OD & $\begin{array}{c}n=68(46 \mathrm{~W} \text { and } 22 \mathrm{M})(34 \text { per G) } \\
\text { Mean age (MA): } 59.6 \pm 27.4 \mathrm{~kg} . \\
\text { Symptom duration: } 1-56 \text { years } \\
\text { with mean of } 5.1 .\end{array}$ & $\begin{array}{c}\text { Grupo A(GA) } \\
\text { (intervention) } \rightarrow \text { TTNS } \\
\text { Grupo B (GB) (control) } \rightarrow \\
\text { PTNS }\end{array}$ & 12 weeks & $\begin{array}{l}\text { Online 1:1 Randomization } \\
\text { Sequence“Sealed } \\
\text { Envelope Lt. 2015" }\end{array}$ \\
\hline $\begin{array}{c}\text { Scaldazza C.V., et al., } \\
2017 \text { [18] }\end{array}$ & $\mathrm{RCT}$ & W with $\mathrm{OAB}$ & $\begin{array}{c}n=60 \mathrm{~W}(30 \text { per } \mathrm{G}) \\
\text { MA: } 58.5 \text { years }(38-72)\end{array}$ & $\begin{array}{c}\mathrm{GA} \rightarrow \text { EMS vaginal }+ \\
\text { exercises } \\
\text { GB } \rightarrow \text { PTNS }\end{array}$ & $1 \mathrm{~m}$ post-treatment. & $\begin{array}{c}\text { Online randomization } \\
\text { GraphPad QuickCalcs } \\
\text { software }\end{array}$ \\
\hline $\begin{array}{c}\text { Preyer O., et al., } 2015 \\
\text { [19] }\end{array}$ & $\begin{array}{c}\text { RCT } \\
\text { Pilot study }\end{array}$ & $\begin{array}{l}\mathrm{W} \text { with OAB, with no } \\
\text { previous PTNS or } \\
\text { anticholinergic treatment }\end{array}$ & $\begin{array}{c}n=36 \mathrm{~W}(18 \text { per } \mathrm{G}) \\
\mathrm{GA}: \mathrm{MA}=57.4 \pm 9.5 \mathrm{~GB}: \\
\mathrm{MA}=55.8 \pm 16.2\end{array}$ & $\begin{array}{c}\text { GA } \rightarrow \text { PTNS } \\
\text { GB } \rightarrow \text { Tolterodine }\end{array}$ & $3 \mathrm{~m}$ (baseline/1-3 m) & $\begin{array}{c}\text { 1:1 adapted } \\
\text { randomization method } \\
\text { (computer assistant) }\end{array}$ \\
\hline $\begin{array}{c}\text { Abulseoud A., et al., } \\
2018 \text { [20] }\end{array}$ & $\mathrm{RCT}$ & $\begin{array}{l}\mathrm{W} \text { with } \mathrm{OAB} \text { and } \mathrm{OD} \text {, and } \\
\text { behavioral treatment failure }\end{array}$ & $\begin{array}{c}n=30 \mathrm{~W}(15 \text { per } \mathrm{G}) \\
\text { G 1: } \mathrm{MA}=48 \pm 16.42 \\
\text { G 2: MA }=48.13 \pm 10.80\end{array}$ & $\begin{array}{c}\text { G 1: TTNS + placebo bottle } \\
\text { identical to G } 2 . \\
\text { G 2: TTNS + Trospium } \\
\text { Chloride }\end{array}$ & 8 Weeks & $\begin{array}{l}\text { 1:1 by random number } \\
\text { table }\end{array}$ \\
\hline $\begin{array}{l}\text { Martín-García M. \& } \\
\text { Cramptom J. } 2019 \text { [3] }\end{array}$ & $\mathrm{RCT}$ & $\begin{array}{l}\mathrm{W} \text { with non-neurogenic OAB } \\
\text { who responded to PTNS } \\
\text { initial treatment of } 12 \text { weeks }\end{array}$ & $\begin{array}{c}n=24 \mathrm{~W}(12 \text { per } \mathrm{G}) \\
\text { G PTNS: } \mathrm{MA}=58 \pm 10 \\
\text { G TTNS: MA }=54 \pm 12\end{array}$ & $\begin{array}{l}\text { Maintenance } \\
\text { G PTNS } \\
\text { G TTNS }\end{array}$ & $\begin{array}{c}6 \mathrm{~m} \\
\text { Evaluation: baseline } \\
\text { (post-treatment with } 12 \\
\text { weeks PTNS), at } 6 \text { weeks, } 3 \\
\mathrm{~m} \text { and } 6 \mathrm{~m}\end{array}$ & $\begin{array}{l}\text { 1:1 via sealed, opaque } \\
\text { envelopes with numbered } \\
\text { sequences }\end{array}$ \\
\hline $\begin{array}{l}\text { Seth J.H., et al., } 2018 \\
\text { [21] }\end{array}$ & $\begin{array}{l}\text { Randomized } \\
\text { pilot trial }\end{array}$ & $\begin{array}{l}\mathrm{M} / \mathrm{W} \text { with } \mathrm{OAB} \text { after } \\
\text { treatment with ineffective } \\
\text { conservative therapy }\end{array}$ & $\begin{array}{c}n=48 \text { (24 multiple sclerosis and } \\
24 \text { Idiopathic OAB) } \\
\text { G } 1 \text { (daily treatment): } \\
\text { MA = 46.4(32-73), } 18 \mathrm{~W} / 6 \mathrm{M}, \\
20 \mathrm{UI} \\
\text { G } 2 \text { (weekly treatment): } \\
\mathrm{MA}=46.9(20-81), 20 \mathrm{~W} / 4 \mathrm{M}, \\
18 \mathrm{UI}\end{array}$ & $\begin{array}{c}\text { TTNS } \\
\text { G 1: } 1 \text { session/day } \\
\text { G 2: } 1 \text { session/week }\end{array}$ & $\begin{array}{c}12 \text { weeks, with evaluation at } \\
4,8 \text { and } 12 \text { weeks }\end{array}$ & $\begin{array}{l}\text { Stratified method by } \\
\text { sealed envelopes }\end{array}$ \\
\hline Welk B., et al., 2020 [22] & $\mathrm{RCT}$ & $\begin{array}{l}\text { W with OAB, neurological } \\
\text { diseases with urinary urgency } \\
\text { with or without UI }\end{array}$ & $\begin{array}{c}n=50 . \text { MA }(73 \%, 22 / 30) ; 10 \% \\
(3 / 30) \text { used spontaneous voiding } \\
\text { catheter } \\
\text { G TTNS } \rightarrow n=26 ; \\
\text { MA = 62 }(54-68) \\
\text { GS } \rightarrow n=24 ; \mathrm{MA}=53(46-64)\end{array}$ & $\begin{array}{c}\text { G TTNS } \rightarrow \text { TTNS. } \\
\text { Increasing amplitude to } \\
\text { maximum tolerance or } \\
\text { flexion of the big toe } \\
\text { GS } \rightarrow \text { TTNS. Constant } \\
\text { amplitude }\end{array}$ & 12 weeks & $\begin{array}{l}1: 1 \text { by random number } \\
\text { generator }\end{array}$ \\
\hline Tudor K.I., et al.020 [23] & $\begin{array}{l}\text { Experimental study of } \\
\text { two groups related } \\
\text { retrospective }\end{array}$ & $\begin{array}{l}\text { Neurological or idiopathic } \\
\text { OAB refractory to 1st line } \\
\text { treatments }\end{array}$ & $\begin{array}{c}n=74(52 \mathrm{~W} / 22 \mathrm{M}), \mathrm{MA}=56.0 \\
(25.2,59.8) . \\
49(66.2 \%) \rightarrow \text { neurogenic OAB } \\
25(33.8 \%) \rightarrow \text { idiopathic OAB }\end{array}$ & PTNS + drug & 12 weeks & Without randomization \\
\hline $\begin{array}{c}\text { Mallman S., et al., } 2020 \\
{[24]}\end{array}$ & $\mathrm{RCT}$ & W with $\mathrm{OAB}$ & $\begin{array}{c}n=50(25 \text { per } \mathrm{G}) \\
\mathrm{MA}=61.48 \pm 10.10\end{array}$ & $\begin{array}{c}\text { G transcutaneous sacral } \\
\text { EMS } \\
\text { G TTNS }\end{array}$ & 6 weeks & $\begin{array}{l}\text { Sequence generated in } 2 \mathrm{C} \\
\text { by WinPEPI version } 11.63\end{array}$ \\
\hline
\end{tabular}


Table 2. Cont.

\begin{tabular}{|c|c|c|c|c|c|}
\hline Author/s & Variables & Results & Conclusions & $\begin{array}{l}\text { Adverse Effects and } \\
\text { Limitations }\end{array}$ & $\begin{array}{c}\text { Met } \\
\text { Quality }\end{array}$ \\
\hline $\begin{array}{l}\text { Ramírez-García I., et al., } \\
2019 \text { [17] }\end{array}$ & $\begin{array}{l}\text { Difference day and night } \\
\text { urination } \\
\text { Mean voiding volume } \\
\text { Number of urgencies and } \\
\text { UI Quality of life }\end{array}$ & $\begin{array}{l}\text { At the beginning/12 weeks. GA: } n=34 / \mathrm{GB}: n=34 \\
\text { Differences by protocol: GA: } n=32 \text { /GB: } n=29 \text {. Post intervention } \\
\text { differences: GA: } n=34 / \mathrm{GB}: n=34 \text {. Difference adjustment with } 95 \% \\
\text { confidence interval } \\
\text { Quality of life I-QoL: GA }(21.5 \mathrm{p}), \mathrm{GB}(22.1 \mathrm{p})(P<0.001)\end{array}$ & $\begin{array}{l}\text { Both techniques improve } \\
\text { symptoms and quality of } \\
\text { life } \\
\text { Treatment adherence } \\
\quad(P=0.236)\end{array}$ & $\begin{array}{l}\text { No serious adverse effects } \\
\text { Study carried out to } \\
\text { measure differences } \\
\text { between groups. High cost. }\end{array}$ & $7 / 10$ \\
\hline $\begin{array}{c}\text { Scaldazza C.V., et al., } \\
2017 \text { [18] }\end{array}$ & $\begin{array}{c}\text { Number of voiding in } \\
24 \mathrm{~h} \\
\text { Number of UUI episodes } \\
\text { Nocturia } \\
\text { Quality of life (OAB-q SF) } \\
\text { Bladder urgency } \\
\text { perception } \\
\text { (PPIU-S) } \\
\text { Perception of global } \\
\text { improvement (PGI-I) }\end{array}$ & $\begin{array}{c}\text { Number of daily voiding } \rightarrow \mathrm{GA} \rightarrow P=0.0620 ; \mathrm{GB} \rightarrow \\
P=0.0307 ; \text { Difference between groups }(\mathrm{DG}) \rightarrow P=0.3758 \\
\text { Number of UUI episodes } \rightarrow \mathrm{GA}: P=0.1293 ; \mathrm{GB} \rightarrow P=0.0009 ; \mathrm{DG}: \\
P=0.0251 \\
\text { Nocturia } \rightarrow \mathrm{GA}: P=0.1683 ; \mathrm{GB} \rightarrow P=0.0201 ; \mathrm{DG}: P=0.049 \\
\text { Voiding volume } \rightarrow \mathrm{GA}: P=0.0048 ; \mathrm{GB} \rightarrow P=0.0003 \text {; DG: } \\
P=0.0222 \\
\text { OAB-q SF: } 6 \text { items: GA: } P=0.0420 ; \mathrm{GB}: P<0.0001 ; \mathrm{DG}: \\
P=0.0172 . \\
\text { 13 items: GA: } P=0.0420 ; \mathrm{GB}: P<0.0001 ; \mathrm{DG}: P=0.0295 \\
\text { PPIU-S: GA: } P=0.1014 ; \mathrm{GB}: P=0.0001 ; \mathrm{DG} P=0.0459 \\
\text { PGI-I } \rightarrow \mathrm{GA}: P=0.0415 ; \mathrm{GB}: P=0.0415\end{array}$ & $\begin{array}{l}\text { PTNS 1st line treatment } \\
\text { (efficacy, minimally } \\
\text { invasive) }\end{array}$ & $\begin{array}{l}\text { Without significant side } \\
\text { effects }\end{array}$ & $6 / 10$ \\
\hline $\begin{array}{c}\text { Preyer O., et al., } 2015 \\
\text { [19] }\end{array}$ & $\begin{array}{l}\text { Differences in number of } \\
\text { voids in } 24 \mathrm{~h} \text { between } \\
\text { both groups (voiding } \\
\quad \text { diaries). } \\
\text { Number of UI episodes } \\
\text { Quality of life (QoL-VAS) }\end{array}$ & $\begin{array}{c}n=16 \text { per group } \\
\text { Number of voids } \rightarrow \text { without significant decrease } 1-3 \mathrm{~m} \text { in both } \mathrm{G} \\
(P=0.13) \text {, DGNS }(P=0.96) \text {. No significant differences in number at } \\
\text { the beginning and post treatment }(P=0.79) \\
\text { Quality of life (QoL-VAS) } \rightarrow \text { depends on the initial values, in GB } \\
\text { mean values are lower than GA in } 1-3 \mathrm{~m} \text { Increase in both } \mathrm{G} 1 \text { and } \\
3 \mathrm{~m} \text {, without significant changes }(P=0.07) \\
\text { Number of UI episodes in } 24 \text { hours } \rightarrow \text { depends on the number } \\
\text { episodes at the beginning of treatment }(P=0.0001) \text {. NSDG pre/post } \\
\text { treatment }(P=0.89) \text {. Significant changes in } 3 \mathrm{~m} \text { (baseline } / 1 \mathrm{~m}) \\
(P=0.03)\end{array}$ & $\begin{array}{l}\text { Both are effective } \\
\text { treatments: decrease in } \\
\text { number of UI episodes, but } \\
\text { not in urinary frequency. } \\
\text { PTNS has fewer side effects. }\end{array}$ & $\begin{array}{l}\text { The first } 4 \text { weeks mainly } \\
\text { GB } \rightarrow \text { dry mouth and } \\
\text { dizziness. } 9 \text { participants } \\
\text { ( } 3 \mathrm{~m}) \\
\text { GA } \rightarrow \text { pain in puncture area. } \\
3 \text { participants }(3 \mathrm{~m}) \text {. } \\
\text { Small sample size and } \\
\text { standard deviations greater } \\
\text { than expected, which could } \\
\text { be due to a type II error; no } \\
\text { blinding }\end{array}$ & $6 / 10$ \\
\hline $\begin{array}{c}\text { Abulseoud A., et al., } \\
2018 \text { [20] }\end{array}$ & $\begin{array}{l}\text { Brief OAB Symptom } \\
\text { Score } \\
\text { (OABSS) } \\
\text { Short form of } \\
\text { incontinence impact } \\
\text { questionnaire } \\
\text { (IIQ-7) }\end{array}$ & 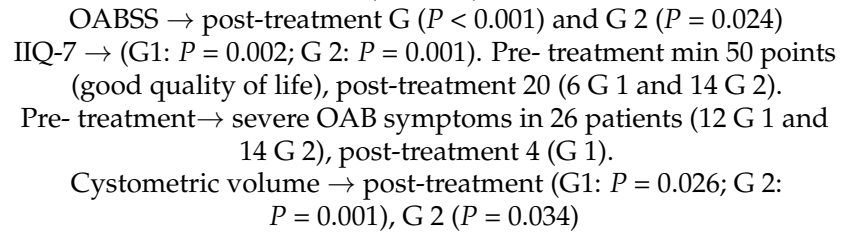 & $\begin{array}{l}\text { TTNS is tolerable and } \\
\text { effective when combined } \\
\text { with Trospium Chloride. } \\
\text { Better results without side } \\
\text { effects. }\end{array}$ & $\begin{array}{l}\text { Without side effects } \\
\text { Limitation: need for another } \\
\text { group with sodium chloride } \\
\text { treatment without TTNS. } \\
\text { Longer follow-up studies } \\
\text { needed. }\end{array}$ & $8 / 10$ \\
\hline
\end{tabular}


Table 2. Cont.

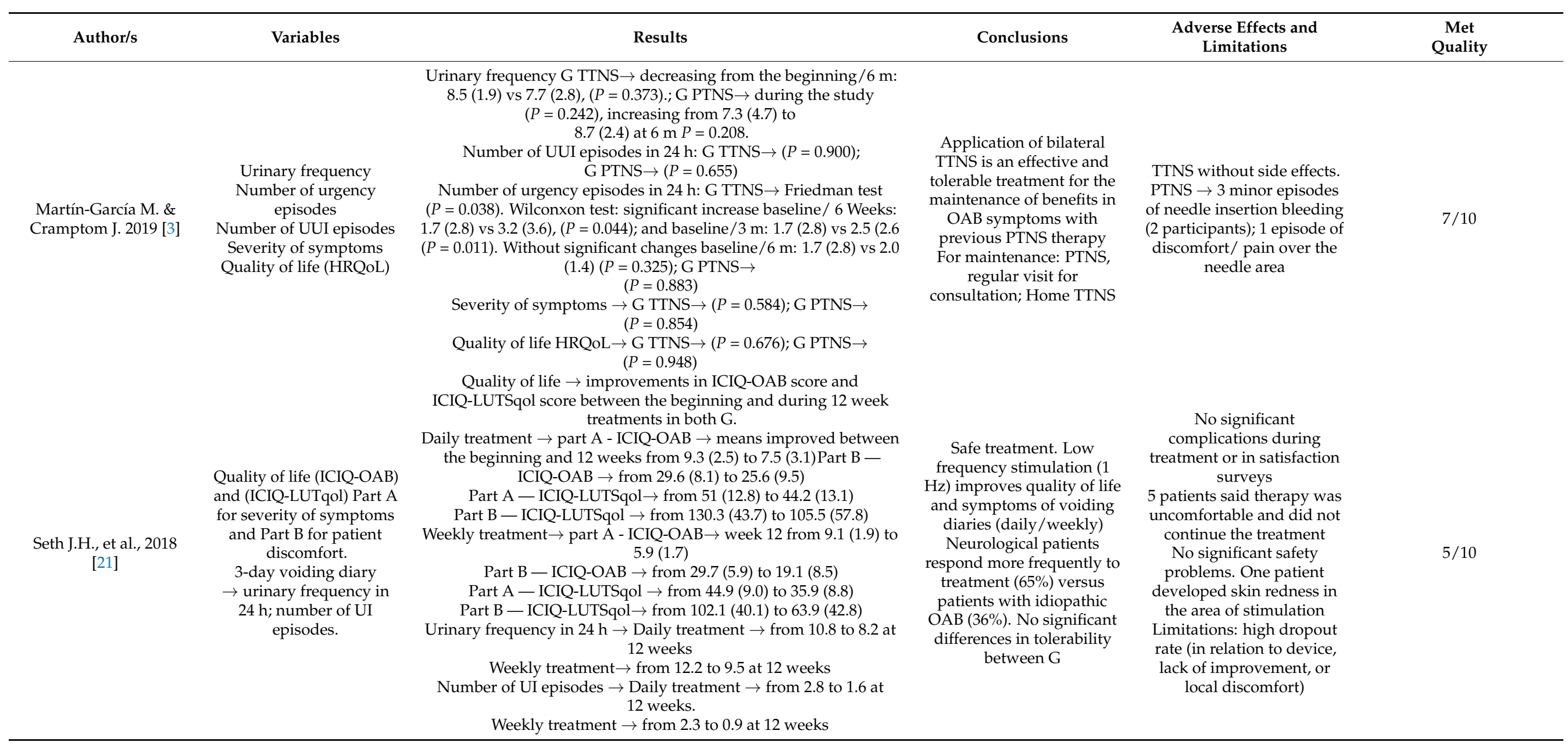


Table 2. Cont.

\begin{tabular}{|c|c|c|c|c|c|}
\hline Author/s & Variables & Results & Conclusions & $\begin{array}{l}\text { Adverse Effects and } \\
\text { Limitations }\end{array}$ & $\begin{array}{c}\text { Met } \\
\text { Quality }\end{array}$ \\
\hline Welk B., et al., 2020 [22] & $\begin{array}{l}\text { Questionnaire } \\
\text { (PPBC) Compress weight } \\
\text { in } 24 \mathrm{~h} \text {, for UI 3-day } \\
\text { voiding diary } \rightarrow \text { Urinary } \\
\text { frequency and functional } \\
\text { capacity in } 24 \mathrm{~h} \text { Quality of } \\
\text { life (OAB-q SF, in G with } \\
\text { OAB) Neurological } \\
\text { patients } \rightarrow \text { (NBSS), } \\
\text { (Qualiveen-SF) }\end{array}$ & $\begin{array}{c}\text { PPBC } \rightarrow 13 \%(3 / 24) \text { of sham patients and } 15(4 / 26) \text { of TTNS } \\
\text { treatment were considered responders }(P=0.77) \text { Marginal mean of } \\
\text { the end of the PPBC score was } 3.3(2.8-3.7) \text { for TTNS vs } 2.9(2.5-3.4) \\
\text { for simulated }(P=0.30) \\
\text { Compress weight in } 24 \mathrm{~h} \rightarrow \mathrm{NSDG}(P=0.64) \\
\text { Functional capacity } \rightarrow \mathrm{NSDG}(P=0.12) \\
\text { Urinary frequency in } 24 \mathrm{~h} \rightarrow \mathrm{NSDG}(P=0.32) \\
\text { OAB-qSF Questionnaire } \rightarrow \text { NSDG in symptom discomfort } \\
(P=0.82) \text { and quality of life }(P=0.29) \\
\text { NBSS } \rightarrow \text { NSDG }(P=0.16) \\
\text { Qualiveen-SF } \rightarrow \text { NSDG }(P=0.85) \\
\text { Global assessment of improvement } \rightarrow \text { NSDG }(P=0.27)\end{array}$ & $\begin{array}{l}\text { TTNS does not display } \\
\text { greater efficacy in patient } \\
\text { perception of OAB } \\
\text { symptoms and objective } \\
\text { parameters evaluated }\end{array}$ & $\begin{array}{c}\text { With no adverse effects } \\
\text { during the study } \\
\text { Limitation in results } \\
\text { generality, most had UI and } \\
\text { had failed with } \\
\text { pharmacological therapy. } \\
\text { Small sample }\end{array}$ & $8 / 10$ \\
\hline $\begin{array}{l}\text { Tudor K.I., } \\
\text { et al., } 2020 \text { [23] }\end{array}$ & $\begin{array}{l}\text { ICIQ-OAB questionnaire } \\
\text { and ICIQ-LUTSqol } \\
\text { 3-day voiding diary } \rightarrow \\
\text { urgency and severity of } \\
\text { UI }\end{array}$ & $\begin{array}{c}64(86 \%) \text { completed } 12 \text { weeks. Significant improvements at } 12 \text { weeks } \\
\text { of treatment in ICIQ-OAB } \\
\text { ICIQ-LUTSqol, change in urinary frequency over } 24 \text { h and severity } \\
\text { of UI in bladder diary } \\
\text { G neurogenic VH } \rightarrow \text { ICIQ-OAB }(P=0.04) \text {; ICIQ-LUTSqol }(P=0.05) \\
\text { [in ICIQ-OAB, odds ratio (IC } 95 \%) 0,93(0,87,0,99), P=0,03] \text {, severity } \\
\text { of UI [in bladder diary, odds ratio (IC } 95 \%) 0.05(0.01,0.63), P=0.02] \\
\text { and QoL [IUTQ-LUTSqol, odds ratio (IC } 95 \%) 0.98(0.96,0.99), \\
P=0.007 \text { ] at } 12 \text { weeks }\end{array}$ & $\begin{array}{c}\text { PTNS is a possible } \\
\text { alternative treatment in } \\
\text { patients with neurological } \\
\text { disease and with ineffective } \\
\text { or intolerable 1st line } \\
\text { treatment }\end{array}$ & $\begin{array}{l}\text { No adverse effects. } 5 \\
\text { patients had mild } \\
\text { discomfort at the needle } \\
\text { insertion area } \\
\text { Lack of blinding, lack of a } \\
\text { placebo or control group, } \\
\text { and lack of urodynamic } \\
\text { assessment before treatment } \\
\text { Not validated } \\
\text { questionnaires in patients } \\
\text { with neurogenic OAB }\end{array}$ & $5 / 10$ \\
\hline $\begin{array}{c}\text { Mallman S., et al., } 2020 \\
\text { [24] }\end{array}$ & $\begin{array}{l}\text { Quality of life: KHQ } \\
\text { Severity of UI: ISI } \\
\text { Discomfort due to OAB } \\
\text { symptoms: OAB-V8 }\end{array}$ & $\begin{array}{c}\text { NSDG }(P>0.005) \\
\text { OAB-V8: (6 weeks } P=0.0019) \mathrm{G} \text { TPNS } / \mathrm{G} \text { transcutaneous sacral } \\
\text { EMS } \\
\text { KHQ e ISI: NSDG }\end{array}$ & $\begin{array}{l}\text { Both therapies are effective } \\
\text { and safe for the treatment of } \\
\text { women with OAB, UUI, } \\
\text { and MUI }\end{array}$ & No side effects & $6 / 10$ \\
\hline
\end{tabular}

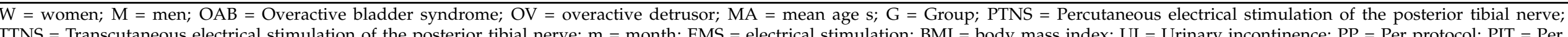

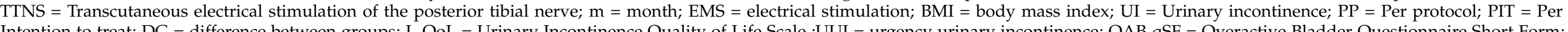

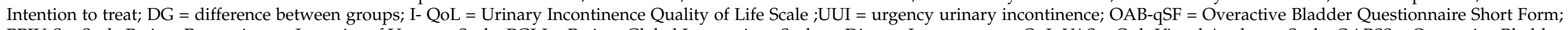

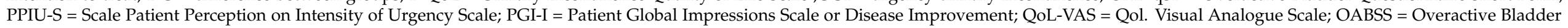

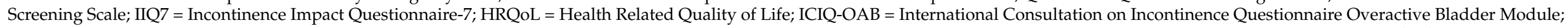

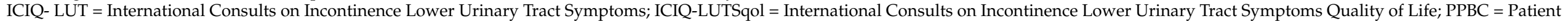

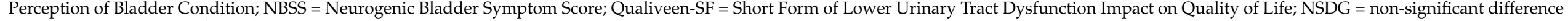
between groups; IC = confidence interval 
Table 3. Characteristics of the observational studies included in the systematic review. Sevilla, ES. 2021.

\begin{tabular}{|c|c|c|c|c|c|c|}
\hline Author/s & Study Design & Study Population & Sample Size & \multicolumn{2}{|c|}{ Intervention } & Follow-Up \\
\hline Salatzki J., et al., 2019 [25] & $\begin{array}{l}\text { Cross-sectional } \\
\quad \text { (cohort) }\end{array}$ & $\begin{array}{l}\text { Positive response to PTNS } \\
\text { treatments (10-12 weeks) }\end{array}$ & $\begin{array}{c}n=83 \text { PTNS-SEQ } \\
\text { G } 1: n=28 \\
\text { G } 2: n=24 \\
\text { G } 3: n=31\end{array}$ & \multicolumn{2}{|c|}{$\begin{array}{c}\text { G } 1 \rightarrow \text { non-responders; no maintenance therapy } \\
\text { G } 2 \rightarrow \text { responders; possibility of maintenance therapy: they } \\
\text { did not do it } \\
\text { G } 3 \rightarrow \text { responders who underwent maintenance therapy }\end{array}$} & 18 weeks \\
\hline Leroux P.A., et al., 2018 [5] & Prospective & $\begin{array}{c}\text { Idiopathic or refractory OAB to } \\
\text { anticholinergic treatment }\end{array}$ & $\begin{array}{c}n=97 \text { treated with TTNS } \\
20(21 \%) \mathrm{M} ; 77(79 \%) \mathrm{W} \\
\mathrm{MA}=58.4 \pm 16.6\end{array}$ & \multicolumn{2}{|c|}{ TTNS } & $24 \mathrm{~m}$ \\
\hline $\begin{array}{l}\text { Moratalla-Charcos L.M., } \\
\text { et al., } 2018 \text { [26] }\end{array}$ & $\begin{array}{l}\text { Pilot study } \\
\text { Prospective }\end{array}$ & $\begin{array}{l}\text { OAB with or without OD/UI, } \\
\text { without success in } \\
\text { pharmacological treatment or } \\
\text { dropout due to adverse effects }\end{array}$ & $\begin{array}{c}n=45: 38 \mathrm{~W} \text { and } 7 \mathrm{M} . \\
\mathrm{MA}=66.6 \pm 10.5(41-83) . \mathrm{OD}: \\
53.3 \%\end{array}$ & \multicolumn{2}{|c|}{ TTNS } & 12 weeks \\
\hline Palmer C., et al 2019 [27] & Retrospective & $\begin{array}{c}>65 \text { with idiopathic OAB, after } \\
\text { treatment with PTNS }\end{array}$ & $\begin{array}{c}n=52: 23 \mathrm{M}(44.3 \%) \\
29 \mathrm{~W}(55.8 \%) \\
\mathrm{MA}=75.75(65 \text { to } 93) \\
\text { BMI }=26.33 \\
(17.4 \text { to } 43.9) \mathrm{kg} / \mathrm{m}^{2}\end{array}$ & \multicolumn{2}{|c|}{ PTNS } & 12 weeks \\
\hline Author/s & Variables & \multicolumn{2}{|c|}{ Results } & Conclusions & $\begin{array}{l}\text { Adverse Effects and } \\
\text { Limitations }\end{array}$ & $\begin{array}{c}\text { Met } \\
\text { Quality }\end{array}$ \\
\hline Salatzki J., et al., 2019 [25] & $\begin{array}{c}\text { ICIQ-OAB } \rightarrow \text { UI in OAB } \\
\text { ICIQ-LUT } \rightarrow \text { OAB } \\
\text { symptoms. } \\
\text { 3-day voiding diary } \\
\text { PTNS-SEQ questionnaire } \rightarrow \\
\text { variables observation to } \\
\text { return to maintenance }\end{array}$ & \multirow{2}{*}{\multicolumn{2}{|c|}{ 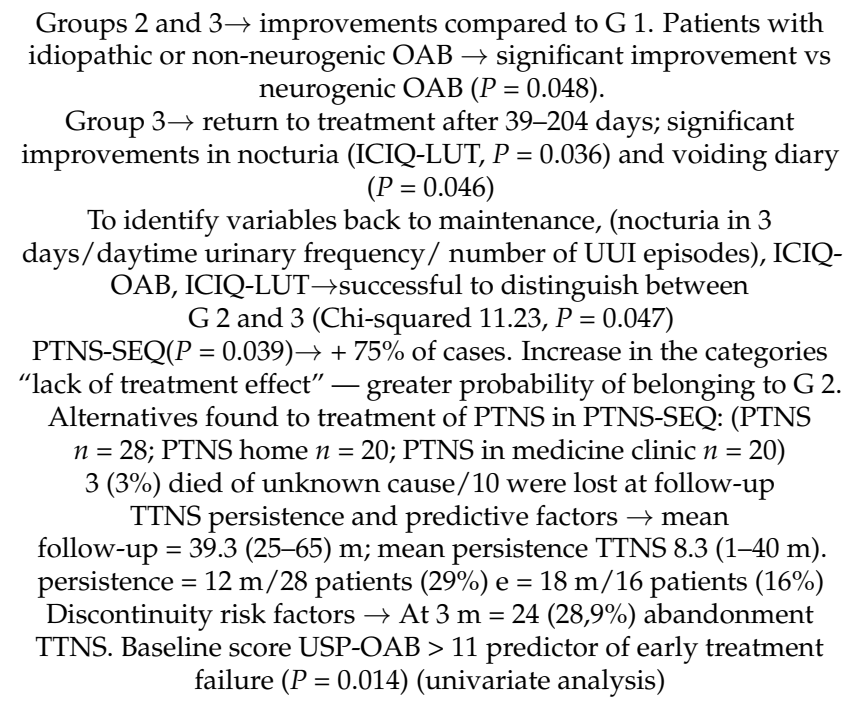 }} & $\begin{array}{l}12 \text { weeks of PTNS } \rightarrow \text { safe } \\
\text { and effective treatment for } \\
\text { OAB. A beneficial response } \\
\text { with PTNS in nocturia was } \\
\text { a factor to return to } \\
\text { maintenance. The voiding } \\
\text { diary offers more objective } \\
\text { results for the evaluation of } \\
\text { the treatment }\end{array}$ & $\begin{array}{l}\text { No side effects } \\
\text { Limitations: small sample } \\
\text { size; difference in number } \\
\text { of participants between G; } \\
\text { results only applicable to } \\
\text { public health. }\end{array}$ & $21 / 22$ \\
\hline Leroux P.A., et al., 2018 [5] & $\begin{array}{c}\text { Questionnaire effectiveness } \\
\text { USP and USP-OAB } \\
\text { Treatment discontinuity and } \\
\text { adverse effects } \\
\text { Comorbidities } \\
\text { Drugs during treatment } \\
\text { follow-up }\end{array}$ & & & $\begin{array}{l}\text { TTNS treatment for } \\
\text { refractory OAB. Few } \\
\text { patients continued } \\
\text { long-term therapy, probably } \\
\text { due to a decrease in efficacy } \\
\text { over time. }\end{array}$ & $\begin{array}{l}\text { No adverse effects or pain } \\
\text { Limitation: loss in } \\
\text { follow-up during the study, } \\
\text { and lack of placebo group, } \\
\text { and objective urodynamic } \\
\text { data }\end{array}$ & $17 / 22$ \\
\hline
\end{tabular}


Table 3. Cont.

\begin{tabular}{|c|c|c|c|c|c|}
\hline Author/s & Variables & Results & Conclusions & $\begin{array}{l}\text { Adverse Effects and } \\
\text { Limitations }\end{array}$ & $\begin{array}{c}\text { Met } \\
\text { Quality }\end{array}$ \\
\hline $\begin{array}{l}\text { Moratalla-Charcos L.M., } \\
\text { et al., } 2018 \text { [26] }\end{array}$ & $\begin{array}{l}\text { 3-day voiding diary } \\
\rightarrow \text { Urinary frequency, } \\
\text { nocturia, number of } \\
\text { urgency episodes, number } \\
\text { of UUI episodes, maximum } \\
\text { voiding volume } \\
\text { OABQ-SF } \\
\text { Subjective improvements } \\
\text { Satisfaction level }\end{array}$ & $\begin{array}{c}n=39 / 45(86.6 \%) \rightarrow \text { completed } 12 \text { weeks treatment } \\
\text { Significant differences before and after treatment }(P<0.05) \text { in } \\
\text { urinary frequency, nocturia, number of urgency episodes, number of } \\
\text { UUI episodes, maximum voiding volume. OABq-SF: } P>0.05 \text {. } \\
\text { Statistically significant differences in GOD vs OAB } \\
\text { Treatment satisfaction } \rightarrow \text { patients with mellitus diabetes } \\
(P=0.043) \text {, in diabetes } \mathrm{W}(P=0.042) \text {. In ordinal regression with } 4 \\
\text { independent variables: number of vaginal deliveries }(P=0.011) ; \\
\text { psychiatric history }(P=0.001) \text { were significant. Group with OD } \rightarrow \\
\text { better satisfaction by increasing the number of vaginal deliveries } \\
\text { and lower satisfaction for } \mathrm{W} \text { and patients with diabetes. In ordinal } \\
\text { regression with } 3 \text { independent variables: number of vaginal } \\
\text { deliveries } \\
(P=0.05) \text { was significant }\end{array}$ & $\begin{array}{l}\text { OAB treatment with TTNS } \\
\text { is an effective, safe, } \\
\text { minimally invasive and } \\
\text { well tolerated therapy. In } \\
\text { this study, all variables } \\
\text { improved significantly } \\
\text { compared to baseline. }\end{array}$ & $\begin{array}{l}\text { Adverse effect: mild pain } \\
\text { on plantar flexion, but no } \\
\text { cases of dermatitis } \\
\text { Limitation: lack of } \\
\text { completion of voiding } \\
\text { diaries, the OABQ-SF } \\
\text { questionnaire, and } \\
\text { subjective improvements, in } \\
\text { addition to lack of a control } \\
\text { group }\end{array}$ & $17 / 22$ \\
\hline Palmer C., et al 2019 [27] & $\begin{array}{c}\text { OAB-V8 } \rightarrow \text { OAB symptoms } \\
\text { 3-day voiding diary } \\
\text { Global impression of } \\
\text { patient satisfaction (GIPS) }\end{array}$ & $\begin{array}{c}n=21(39 \%) \text { used combination therapy during PTNS } \\
\text { After PTNS } \rightarrow 37 \text { patients (70\%) reported symptom improvements; } \\
7 \text { used anticholinergic, } 6 \text { used } B 3 \text { adrenoceptor agonist, } 5 \text { received } \\
\text { intravesical injections of onabotulinumtoxnA, and } 2 \text { underwent } \\
\text { sacral neuromodulation } \\
\text { Mean old age } \rightarrow n=13,1 \text { or } 2 \text { medical comorbidities; } n=10,3 \text {, or } 4 \\
\text { medical comorbidities; } n=6+5 . n=20 \mathrm{~W}(69 \%) \text { used } \\
\text { anticholinergic treatments before PTNS; } n=11 \mathrm{~W}(38 \%) \text { used } \\
\text { combination therapy during PTNS }\end{array}$ & $\begin{array}{l}\text { Effectiveness and viability } \\
\text { of the PTNS technique for } \\
\text { the treatment of OAB in } \\
\text { elderly patients is observed, } \\
\text { being able to choose as a } \\
\text { 2nd line treatment. } \\
\text { Decrease in the use of } \\
\text { anticholinergics by PTNS } \\
\text { therapy }\end{array}$ & $\begin{array}{l}\text { Retrospective descriptive } \\
\text { study, small sample size } \\
\text { which could influence the } \\
\text { results. More objective } \\
\text { measures should have been } \\
\text { used to determine the } \\
\text { success of the treatment, } \\
\text { such as voiding diary } \\
\text { parameters or urodynamic } \\
\text { parameters }\end{array}$ & $13 / 22$ \\
\hline
\end{tabular}

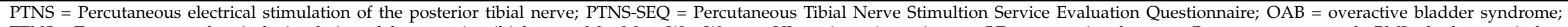

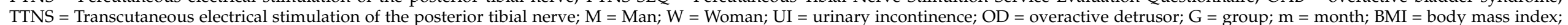

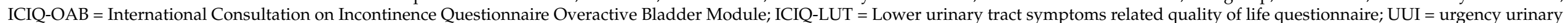

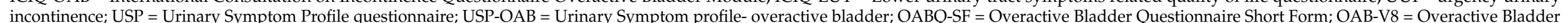

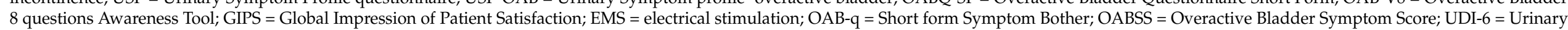

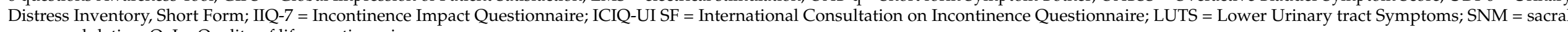
neuromodulation; $\mathrm{QoL}=$ Quality of life questionnaire. 
Table 4. Characteristics of the systematic reviews and meta-analyses included in the systematic review. Sevilla, ES. 2021.

\begin{tabular}{|c|c|c|c|c|c|c|c|}
\hline Author/s & $\begin{array}{c}\text { Study } \\
\text { Design }\end{array}$ & $\begin{array}{c}\text { Number and Design } \\
\text { of Studies }\end{array}$ & Study Participants & Inerventions & Variables & Results & Conclusions and Limitations \\
\hline $\begin{array}{l}\text { Wibison E., et al., } \\
2015 \text { [28] }\end{array}$ & $\begin{array}{l}\text { Meta- } \\
\text { analysis }\end{array}$ & $\begin{array}{c}16 \text { studies } \\
11 \text { RCTs } \\
5 \text { non-comparative } \\
\text { prospective studies }\end{array}$ & $\begin{array}{c}\text { Participants with } \\
\text { non-neurogenic OAB } \\
n=787 \\
480 ; \text { PTNS } \\
\text { 108; antimuscarinics } \\
63 \text { combined therapy } \\
136 \text { sham or placebo } \\
\text { treatment } \\
\text { More } \mathrm{W} \text { than } \mathrm{M} \\
(10 / 16 \mathrm{~W})\end{array}$ & $\begin{array}{c}\text { PTNS vs sham treatment } \\
\text { PTNS vs antimuscarinics } \\
\text { PTNS in non-comparative } \\
\text { studies }\end{array}$ & $\begin{array}{l}\text { Percentage of } \\
\text { responders or patients } \\
\text { with positive response } \\
\text { Voiding diary } \\
\text { parameters (urinary } \\
\text { frequency; nocturia; UI } \\
\text { and voiding volume) }\end{array}$ & $\begin{array}{l}\text { PTNS vs sham } \\
\text { procedure } \\
\text { Urinary frequency and } \\
\text { UI episodes } \\
\text { PTNS vs } \\
\text { antimuscarinics } \\
\text { PTNS in comparative } \\
\text { studies }\end{array}$ & $\begin{array}{l}\text { PTNS therapy is effective for the } \\
\text { short-term treatment of OAB, with } \\
\text { greater efficacy than with sham } \\
\text { treatment, and comparable with } \\
\text { antimuscarinic drugs (but with } \\
\text { fewer adverse effects). However, } \\
\text { multimodal therapy was found to be } \\
\text { more effective } \\
\text { PTNS could be a maintenance } \\
\text { therapy due to its safety and } \\
\text { durability } \\
\text { Dose, duration, frequency, pulse of } \\
\text { PTNS, duration of study follow-up } \\
\text { and demographic characteristics of } \\
\text { the subjects were highly variable in } \\
\text { the studies included }\end{array}$ \\
\hline $\begin{array}{l}\text { Booth J., et al., } \\
2018 \text { [4] }\end{array}$ & $\begin{array}{l}\text { Systematic } \\
\text { review }\end{array}$ & $\begin{array}{c}13 \text { articles } \\
10 \text { RCTs } \\
3 \text { prospective studies }\end{array}$ & $\begin{array}{c}>18 \text { years old with OAB } \\
\text { with MUI } \\
n=629 \rightarrow 473(70 \%) \mathrm{W} \\
\text { and } 176(28 \%) \mathrm{M}, 16 \\
(2 \%) \text { gender is unknown } \\
36(18 \%) \rightarrow \text { sham } \\
\text { treatment } \\
142(56 \%) \\
\rightarrow \text { anticholinergic; } 26 \\
(10 \%) \rightarrow \text { pelvic floor and } \\
\text { bladder training } \\
9(4 \%) \rightarrow \text { sacral EMS or } \\
\text { without treatment }\end{array}$ & $\begin{array}{l}\text { Durability: } 4-12 \text { weeks (mean: } \\
7.6 \pm 3.6 \text { ). Total number of } \\
\text { sessions } 5-90 \\
\text { (mean } 21.6 \pm 2.3 \text { ) } \\
30 \text { min/individual session, } \\
\text { except } 3 \text { of } 20 \text { min. } \\
3 \text { studies with daily } \\
\text { stimulation, } 7 \text { studies with } \\
2 \text { times / week and } \\
2 \text { studies with } 1 \text { time/week }\end{array}$ & $\begin{array}{c}\text { Urinary urgency } \\
\text { symptoms } \\
\text { Urinary frequency } \\
\text { Nocturia } \\
\text { Number of UI episodes } \\
\text { Quality of life } \\
\text { Adverse effects } \\
\text { Urodynamic changes }\end{array}$ & $\begin{array}{c}\text { Changes in voiding } \\
\text { diary } \\
\text { Score in OAB symptoms } \\
\text { Effectiveness of TTNS } \\
\text { Observational studies } \rightarrow \\
3 / 3 \\
\text { General combined } \\
\text { result } \rightarrow 9 / 13\end{array}$ & $\begin{array}{l}\text { All studies observed improvements } \\
\text { with TTNS treatment. It is safe and } \\
\text { tolerable, due to this factor, its low } \\
\text { cost, its ease of application and the } \\
\text { possibility of self-administration by } \\
\text { the patient, more studies are } \\
\text { necessary to show its use as a 2nd } \\
\text { line treatment }\end{array}$ \\
\hline $\begin{array}{l}\text { Tutolo M., et al., } \\
\quad 2018 \text { [29] }\end{array}$ & $\begin{array}{l}\text { Systematic } \\
\text { review }\end{array}$ & 9 articles, all RCTs & $\begin{array}{c}\text { Patients with OAB } \\
\text { treated by SNM or } \\
\text { PTNS }\end{array}$ & $\begin{array}{c}\text { SNM and PTNS } \\
\text { PTNS: } 4 \text { RCTs } \rightarrow 388 \text { patients } \\
\text { PTNS vs Tolterodine } \rightarrow 3 \mathrm{~m} \\
(94 \% \mathrm{~W}) \\
\text { PTNS vs sham therapy } \rightarrow 3 \mathrm{~m} \\
\text { PTNS vs placebo } \rightarrow 12 \text { weeks } \\
\text { PTNS vs vaginal electrical } \\
\text { stimulation } \rightarrow 12 \text { weeks }\end{array}$ & $\begin{array}{c}\text { Number of UI episodes } \\
\text { and severity } \\
\text { Number of compress in } \\
24 \mathrm{~h} \\
\text { Urinary frequency } \\
\text { Voiding volume } \\
\text { Urinary urgency }\end{array}$ & $\begin{array}{l}\text { PTNS efficacy } \\
\text { PTNS safety }\end{array}$ & $\begin{array}{l}\text { There are no high-quality studies } \\
\text { able to guide professionals to choose } \\
\text { between different treatments. This } \\
\text { study shows that sacral stimulation } \\
\text { and PTNS are safe and effective. } \\
\text { SNM has more long-lasting effects, } \\
\text { while PTNS needs to have } \\
\text { maintenance treatment } \\
\text { Limitations: number of results due } \\
\text { to the impossibility of evaluator and } \\
\text { patient blinding in the studies }\end{array}$ \\
\hline
\end{tabular}


Table 4. Cont.

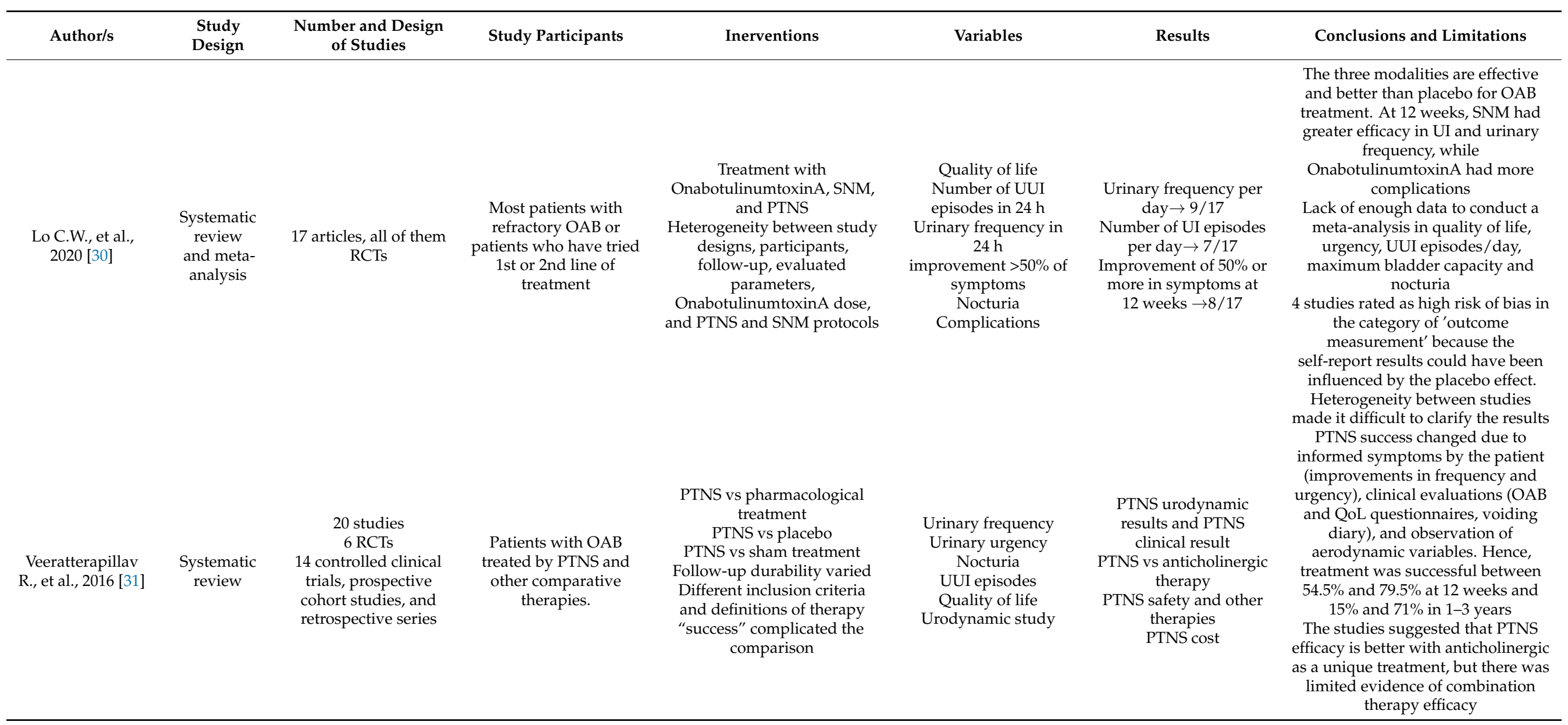


Table 4. Cont.

\begin{tabular}{|c|c|c|c|c|c|c|c|}
\hline Author/s & $\begin{array}{l}\text { Study } \\
\text { Design }\end{array}$ & $\begin{array}{c}\text { Number and Design } \\
\text { of Studies }\end{array}$ & Study Participants & Inerventions & Variables & Results & Conclusions and Limitations \\
\hline $\begin{array}{l}\text { Wang M., et al., } \\
2020 \text { [32] }\end{array}$ & $\begin{array}{l}\text { Systematic } \\
\text { review } \\
\text { and meta- } \\
\text { analysis }\end{array}$ & $\begin{array}{c}28 \text { articles, } 12 \text { RCTs } \\
16 \text { observational } \\
\text { studies }\end{array}$ & $\begin{array}{l}246 \text { patients with OAB } \\
\text { symptoms treated by } \\
\text { PTNS and other } \\
\text { comparative therapies }\end{array}$ & $\begin{array}{l}30 \text { min PTNS for } 12 \text { weeks in } \\
6 / 2 \text { studies, the rest of studies } \\
\text { had different protocols } \\
\text { PTNS vs Tolterodine } \\
\text { PTNS vs PTNS + sham } \\
\text { treatment } \\
\text { PTNS vs TTNS }\end{array}$ & $\begin{array}{l}\text { 3-day voiding diary } \\
\text { (urinary frequency/day, } \\
\text { nocturia/day, number } \\
\text { of UUI episodes/day, } \\
\text { number of UI episodes, } \\
\text { daytime urination } \\
\text { frequency/day, voiding } \\
\text { volume and } \\
\text { urodynamic data) } \\
\text { Response rate }\end{array}$ & $\begin{array}{c}\text { Urinary frequency/day } \\
\rightarrow 10 / 28 . \\
\text { Nocturia/día } \rightarrow 13 / 28 \\
\text { Number of UUI and UI } \\
\text { episodes } / \text { day } \rightarrow 8 / 28 \\
\text { and } 10 / 28 \\
\text { Urination frequency } \\
\quad / \text { day } \rightarrow 7 / 28 \\
\text { Voiding volume / day } \\
\rightarrow 8 / 28 \\
\text { Urodynamic data } \\
\text { Treatment response rate } \\
\rightarrow 17 / 28 \\
\text { Comparison with other } \\
\text { therapies }\end{array}$ & $\begin{array}{l}\text { PTNS therapy was shown to be } \\
\text { effective and safe for OAB treatment } \\
\text { Limitations: heterogeneity of the } \\
\text { studies included, however, a } \\
\text { subgroup analysis was performed to } \\
\text { observe that this factor was due to } \\
\text { the study design. Second, evaluation } \\
\text { of the improvements and success of } \\
\text { the variable was done unconsciously } \\
\text { Severe side effects: the most } \\
\text { common was pain in the puncture } \\
\text { area }\end{array}$ \\
\hline
\end{tabular}

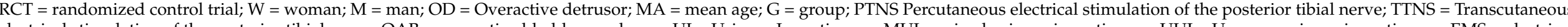

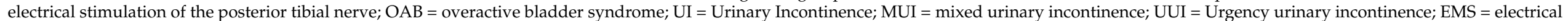
stimulation; $\mathrm{SNM}=$ sacral neuromodulation . 


\subsection{Summary of the Evidence}

As for the comparison between PTNS and TTNS therapy, studies were found $[3,17]$ in which significant changes were observed in the variables of diurnal frequency of urination, nocturnal frequency of urination, $24-\mathrm{h}$ voiding frequency, mean voided volume, and number of episodes of UI and UUI in $24 \mathrm{~h}$ [17].

When TTNS combined with trospium chloride [20] was compared to placebo, a decrease in frequency of urination was observed in both groups $(p=0.001$ and $p=0.003$, respectively); as to mean voided volume, significant improvements were observed in both groups, with greater significance in the combined therapy $(p=0.005)$, although there was a significant delay in the combined therapy group with regard to the first sensation of a full bladder [20].

In other studies [23,32], upon combining PTNS with drugs, 35/53 participants completed the satisfaction survey after treatment, $66 \%$ of whom preferred to continue with maintenance treatment, with a mean interval of 44.4 days (7-155 days) and frequency of sessions of 1.1 months; attendance was observed if there were symptoms of $\mathrm{OAB}$, while patients with multiple sclerosis had the possibility of returning [23].

Following this comparative line, one review was found [4] in which TTNS was compared with diverse therapies, including $3 / 10$ studies that compared simulated therapy, $4 / 10$ anticholinergic, $1 / 10$ exercise, $1 / 10$ behavioral, and $1 / 10$ two different stimulation sites. The three remaining studies compared TTNS with other treatments: extendedrelease oxybutynin vs. TTNS + fármacoM; TTNS vs. transcutaneous sacral foramina vs. combination of the two; bladder and pelvic floor training vs. TTNS.

By contrasting daily or weekly treatment [21] with TTNS, $100 \%$ of weekly participants completed the compliance and experience questionnaire, in comparison to $90.5 \%$ of patients on daily therapy [21]. Although 53\% (18) gave as a result a moderate or significant improvement in symptoms for the global response assessment (GRA), 75\% (13/20) of neurological patients with $\mathrm{OAB}$ and $36 \%(5 / 14)$ of patients with idiopathic $\mathrm{OAB}$ responded to the intervention [21].

With respect to adherence to treatment [5,17] with TTNS, one of the studies [5] established different reasons for discontinuity (in 70 participants): lack of symptom relief (70\%); difficulty in complying (6\%); becoming asymptomatic (8\%). However, $16.9 \%$ (14) of patients continued treatment, with a mean follow-up of 39.3 months [5].

Meanwhile, a BMI of obesity $\left(=30 \mathrm{~kg} / \mathrm{m}^{2}\right)$ was observed to be the only statistically significant variable predictive of failure in the response to PTNS $(p=0.002)$ [27]. Notably, after PTNS therapy, 66\% (19/29) of participants informed of an improvement in their symptoms [27].

Some of the additional complications to those observed in the analysis of results $[3,19,21,23,26,28,30,32]$ were urinary tract infections in 10/17 studies (peer comparisons revealed that OnabotulinumtoxinA was associated with a greater incidence of urine infections vs. placebo, sacral neurostimulation (SNS), and PTNS); ranking in order of fewest infections: first PTNS, second SNS, third placebo, and fourth OnabotulinumtoxinA. Further, urine retention with a need for intermittent catheterization was found in 11/17, with peer comparisons showing that OnabotulinumtoxinA was associated with a greater incidence of retention vs. placebo, SNS, and PTNS; ranking in order of lowest incidence: first SNM, second placebo, third PTNS, and fourth OnabotulinumtoxinA [30].

\section{Discussion}

The aim of this systematic review was to analyze the scientific evidence on the treatment of OAB with UI through procedures of PTNS, compared to TTNS, of the PTN. Nineteen studies were included, which analyze, observe, and compare these therapies with other methods, such as simulated treatment, placebo, anticholinergic or other drugs, sacral electrical stimulation, or vaginal electrical stimulation.

Among the studies whose intervention was based mainly on PTNS or TTNS therapy vs. another therapy, UI presented significant improvement when compared to placebo or 
simulated treatment $[28,29,31]$. Abulseoud A et al. [20] showed a significant improvement in the number of episodes of UI in combined groups of TTNS and trospium choloride compared to TTNS for eight weeks [20]. It is worth noting the significant improvements observed in the review by Veeratterapillay $R$ et al. [31] in UUI after 12 weeks of treatment and two years of maintenance with PTNS therapy, unlike what was observed by Welk B et al. [22] in their RCT with PTNS therapy, with no significant differences between TTNS treatment compared to simulated therapy.

It is worth highlighting the improvements in UI reflected in the systematic review conducted by Booth J et al. [4], when combining TTNS therapy with pelvic floor exercises or behavioral treatment, as well as the results observed in the systematic review and metaanalysis performed by Wang $\mathrm{M}$ et al., as regards the reduction in the number of episodes of UI and UUI per day through PTNS therapy [32].

Apart from two studies analyzed [5,24], parameters related to frequency of urination, urgency of urination, and nocturia, as well as other symptoms of OAB such as voiding volume and urodynamic changes were included as variables and presented dissimilar results between studies.

In a recent study [10] from 2020, significant improvements were observed in the perception of quality of life of patients treated with TTNS and PTNS, with no differences between treatments [10].

By focusing on the quality of life observed in the studies analyzed, it is worth highlighting that all the experimental studies included provided data on this. Some of these studies $[17,18,21,23]$ showed significant improvements in quality of life, through diverse questionnaires, after treatment with PTNS or TTNS, revealing that this improvement increased when TTNS was combined with trospium chloride, although the difference was not significant [20].

In 2013, Peters KM et al. [11] observed improvements in the quality of life of patients with $\mathrm{OAB}$ who were treated with PTNS, evaluated three years after treatment. In the present review, PTNS has been seen to present significant differences in quality of life when compared to vaginal electrostimulation [18], and it has been observed that there are significant differences in increased quality of life in both neurogenic and non-neurogenic OAB [23].

As for other parameters, it is worth noting the RCT of de Scaldazza CV et al. [18] in which significant differences were revealed in terms of the patient's global perception in favor of the PTNS technique compared to vaginal electrostimulation.

Leroux PA et al. [5] in their study showed some of the reasons why there is discontinuity in treatment with TTNS therapy, the most prevalent of which, in $70 \%$ of cases, was sufficient relief from symptoms, while in $6 \%$ it was due to complications for compliance with the treatment, and in $8 \%$ it was due to a complete reduction of the symptoms and becoming asymptomatic [5].

Most of the studies included in this review report of the absence of adverse effects during treatment $[5,17,18,20,22,24,25]$. Studies that combine PTNS and TTNS therapy [17], and those in which TTNS therapy is involved $[5,20,22,25]$, point out that there are no adverse effects after the use of this therapy, except for the study conducted by MoratallaCharcos LM et al. [26], who speak of mild pain on plantar flexion after the use of this technique.

Regarding PTNS therapy, no serious adverse effects were found, only minor bleeding episodes were mentioned or mild discomfort at the needle insertion site $[3,25,29]$, sometimes causing hematomas or paresthesia at the puncture site [31].

As for the electrical stimulation parameters with PTNS therapy, most studies referred to weekly sessions for 12 weeks as the time of treatment. Although some of them did not show the other parameters, the rest coincided with regard to sessions of 30 min duration, a frequency of $20 \mathrm{~Hz}$, pulse of $200 \mathrm{~ms}$, 34-gauge needle inserted approximately five $\mathrm{cm}$ above the medial malleolus, and electrode in ipsilateral calcaneus $[17,19,22,23,27]$. In terms 
of amplitude, this was increased to the level of discomfort of the patient, feeling of tickling on the sole of the foot, or flexion of the big toe.

Upon referring to treatment with TTNS therapy, the parameters between the studies are more variable: some of the studies mentioned the same stimulation parameters as those of PTNS therapy, while others varied in terms of frequency, using a frequency of $10 \mathrm{~Hz}$, as was the case of the randomized clinical trial of Welk B et al. [22], also highlighting the frequency of weekly sessions, with a total of three weekly sessions for 12 weeks.

The experimental studies of Abulseoud A et al. [20] and Seth JH et al. [21] stand out due to the use of different parameters, with the former [20] using frequencies of $10 \mathrm{~Hz}$, pulse of $250 \mathrm{~ms}$, treatment three times a week for eight weeks, and with a stimulation time of $30 \mathrm{~min}$. Meanwhile, in the latter study [21], they used amplitudes of $27 \mathrm{~mA}$, pulse between 70 and $560 \mathrm{~ms}$, which varied depending on patient tolerability, for 12 weeks, both daily and weekly. Mallman S et al. [24], in their RCT, showed a stimulus duration of $20 \mathrm{~min}$ with a follow-up of six weeks and a pulse duration of $300 \mathrm{~ms}$.

\section{Conclusions}

It is complicated to be able to establish which electrical stimulation therapy of the PTN is the most effective for treatments of idiopathic OAB with UI in adults, as far as the different parameters observed in this review are concerned, due to the variability of the results obtained and the electrical stimulation parameters used in the studies included. Nevertheless, it is worth highlighting the advantages TTNS therapy presents with respect to PTNS therapy, as this could be more comfortable for the patient, all things being equal in the results variable.

Author Contributions: Conceptualization, A.A.-G. and M.A.-C.; methodology, A.A.-G., M.A.-C., and I.E.-P.; formal analysis, A.A.-G., I.E.-P., and M.A.-C.; investigation, A.A.-G., A.M.P.-L., M.B.-D., and M.J.C.-H.; writing-original draft preparation, A.A.-G. and M.A.-C.; writing-review and editing, A.A.-G. and I.E.-P.; visualization, A.M.P.-L., M.B.-D., and M.J.C.-H. All authors have read and agreed to the published version of the manuscript.

Funding: This research received no external funding.

Institutional Review Board Statement: Not applicable.

Informed Consent Statement: Not applicable.

Conflicts of Interest: The authors declare no conflict of interest.

\section{References}

1. ICD-11-Mortality and Morbility Statistics. Available online: https://icd.who.int/browse11/l-m/en (accessed on 12 July 2021).

2. Kobashi, K.; Nitti, V.; Margolis, E.; Sand, P.; Siegel, S.; Khandwala, S.; Newman, D.; MacDiarmid, S.A.; Kan, F.; Michaud, E. A Prospective Study to Evaluate Efficacy Using the Nuro Percutaneous Tibial Neuromodulation System in Drug-Naïve Patients with Overactive Bladder Syndrome. Urology 2019, 131, 77-82. [CrossRef]

3. Martin-Garcia, M.; Crampton, J. A single-blind, randomized controlled trial to valuate the effectiveness of transcutaneous tibial nerve stimulation (TTNS) in Overactive Bladder symptoms in women responders to percutaneous tibial nerve stimulation (PTNS). Physiotherapy 2019, 105, 469-475. [CrossRef] [PubMed]

4. Booth, J.; Connelly, L.; Dickson, S.; Duncan, F.; Lawrence, M. The effectiveness of transcutaneous tibial nerve stimulation (TTNS) for adults with overactive bladder syndrome: A systematic review. Neurol. Urodyn. 2018, 37, 528-541. [CrossRef]

5. Leroux, P.A.; Brassart, E.; Lebdai, S.; Azzouzi, A.R.; Bigot, P.; Carrouget, J. Transcutaneous tibial nerve stimulation: 2 years follow-up outcomes in the management of anticholinergic refractory overactive bladder. World J. Urol. 2018, 36, 1455-1460. [CrossRef]

6. Angulo, J.C.; Brenes, F.J.; Lizarraga, I.; Rejas, J.; Trillo, S.; Ochayta, D.; Arumi, D. Impacto del número de episodios diarios de incontinencia de urgencia en los resultados descritos por el paciente con vejiga hiperactiva. Actas Urol. Esp. 2016, 40, 173-182. [CrossRef]

7. Milsom, I.; Coyne, K.S.; Nicholson, S.; Kvasz, M.; Chen, C.I.; Wein, A.J. Global prevalence and economic burden of urgency urinary incontinence: A systematic review. Eur. Urol. 2014, 65, 79-95. [CrossRef]

8. Raju, R.; Linder, B.J. Evaluation and treatment of Overactive Bladder in Women. In Mayo Clinic Proceedings; Elsevier LTD: Amsterdam, The Netherlands, 2020; Volume 95, pp. 370-377. 
9. Valles-Antuña, C.; Pérez-Haro, M.L.; González-Ruiz de, L.C.; Quintás-Blanco, A.; Tamargo-Díaz, E.M.; García-Rodríguez, J.; San Martín-Blanco, A.; Fernandez-Gomez, J.M. Estimulación transcutánea del nervio tibial posterior en el tratamiento de la incontinecia urinaria de urgencia refractaria, de origen idiopático y neurogénico. Actas Urol. Esp. 2017, 41, 465-470. [CrossRef]

10. Sousa-Fraguas, M.C.; Lastra-Barreira, D.; Blanco-Díaz, M. Neuromodulación periférica en el síndrome de vejiga hiperactiva en mujeres: Una revisión). Actas Urol. Esp. 2020, 45, 177-187. [CrossRef]

11. Peters, K.M.; Carrico, D.J.; Wooldridge, L.S.; Miller, C.J.; MacDiarmid, S.A. Percutaneous tibial nerve stimulation for the long-term treatment of overactive bladder: 3-year results of the STEP study. J. Urol. 2013, 189, 2194-2201. [CrossRef]

12. Moher, D.; Liberati, A.; Tetzlaff, J.; Altman, D.G.; Grupo PRISMA. Preferred Reporting Items for Systematic Reviews and Meta-Analyses: The PRISMA Statement. Rev. Esp. Nutr. Hum. Diet. 2014, 18, 172-181. [CrossRef]

13. Methley, A.M.; Campbell, S.; Chew-Graham, C.; McNally, R.; Cherghi-Sohi, S. PICO, PICOS and SPIDER: A comparison study of specificity and sensitivity in three search tools for qualitative systematic reviews. BMC Health Serv. Res. 2014, 14, 579. [CrossRef]

14. Maher, C.G.; Sherrington, C.; Herbert, R.D.; Mosely, A.M.; Elkins, M. Reliability of the PEDro scale for rating quality of randomized controlled trials. Phys. Ther. 2003, 83, 713-721. [CrossRef]

15. Argimon Pallás, J.M.; Jimenez Villa, J. Metodos de Investigación Clinica y Epidemiológica, 3rd ed.; Elsevier: Barcelona, Spain, 2004.

16. Vandenbroucke, J.P.; von Elm, E.; Altman, D.G.; Gøtzsche, P.C.; Mulrow, C.D.; Pocock, S.J.; Poole, C.; Schlesselman, J.J.; Egger, M.; Strobe Initiative. Strengthening the Reporting of Observational Studies in Epidemiology (STROBE): Explanation and elaboration. PLoS Med. 2007, 4, 1628-1654. [CrossRef] [PubMed]

17. Ramirez-García, I.; Blanco-Ratto, L.; Kauffmann, S.; Carralero-Martínez, A.; Sánchez, E. Efficacy of transcutaneous stimulation of the posterior tibial nerve compared to percutaneous stimulation in idiopathic overactive bladder syndrome: Randomized control trial. Neurourol. Urodyn. 2019, 38, 261-268. [CrossRef]

18. Scaldazza, C.V.; Morosetti, C.; Giampieretti, R.; Lorenzetti, R.; Baroni, M. Percutaneous tibial nerve stimulation versus electrical stimulation with pelvic floor muscle training for overactive bladder syndrome in women: Results of a randomized controlled study. Int. Braz. J. Urol. 2017, 43, 121-126. [CrossRef] [PubMed]

19. Preyer, O.; Umek, W.; Laml, T.; Bjelic-Radisic, V.; Gabriel, B.; Mittlboeck, M.; Hanzal, E. Percutaneous tibial nerve stimulation versus tolterodina for overactive bladder in women: A randomized controlled trial. Eur. J. Obstet. Gynecol. Reprod. Biol. 2015, 191, 51-56. [CrossRef]

20. Abulseoud, A.; Moussa, A.; Abdelfattah, G.; Ibrain, I.; Saba, E.; Hassouna, M. Transcutaneous posterior tibial nerve electrostimulation with low dose trospium chloride: Could it be used as a second line treatment of overactive bladder in females. Neurourol. Urodyn. 2018, 37, 842-848. [CrossRef] [PubMed]

21. Seth, J.H.; Gonzales, G.; Haslam, C.; Pakzad, M.; Vashisht, A.; Sahai, A.; Knowles, C.; Tucker, A.; Panicker, J. Feasibility of using a novel non-invasive ambulatory tibial nerve stimulation device for the home-based treatment of overactive bladder symptoms. Transl. Androl. Urol. 2018, 7, 912-919. [CrossRef] [PubMed]

22. Welk, B.; Mckibbon, M. A randomized, controlled trial of transcutaneous tibial nerve stimulation to treat overactive bladder and neurogenic bladder patients. Can. Urol. Assoc. J. 2020, 14, 297-303. [CrossRef] [PubMed]

23. Tudor, K.I.; Seth, J.H.; Liechti, M.D.; Ochulor, J.; Gonzales, G.; Haslm, C.; Fox, Z.; Pakzad, M.; Panicker, J.N. Outcomes following percutaneous tibial nerve stimulation (PTNS) treatment for neurogenic and idiopathic overactive bladder. Clin. Auton. Res. 2020, 30, 61-67. [CrossRef] [PubMed]

24. Mallmann, S.; Ferla, L.; Rodrigues, M.P.; Paiva, L.L.; Sanches, P.R.S.; Ferreira, C.F.; Ramos, J.G.L. Comparison of parasacral transcutaneous electrical stimulation and transcutaneous posterior tibial nerve stimulation in women with overactive bladder syndrome: A randomized clinical trial. Eur. J. Obstet. Gynecol. Reprod. Biol. 2020, 250, 203-208. [CrossRef] [PubMed]

25. Salatzki, J.; Liechti, M.D.; Spanudakis, E.; Gonzalez, G.; Baldwin, J.; Haslam, C.; Pakzad, M.; Panicker, J.N. Factors influencing return for maintenance treatment with percutaneous tibial nerve stimulation for the management of the overactive bladder. $B J U$ Int. 2019, 123, 20-28. [CrossRef] [PubMed]

26. Moratalla Charcos, L.M.; Planelles Gómez, J.; García Mora, B.; Santamaria Navarro, C.; Vidal Moreno, J.F. Efficacy and satisfaction with transcutaneous electrostimulation of the posterior tibial nerve in overactive bladder syndrome. J. Clin. Urol. 2018, 11, 331-338. [CrossRef]

27. Palmer, C.; Farhan, B.; Nguyen, N.; Ghoniem, G. Clinical outcomes of percutaneous tibial nerve stimulation in elderly patients with overactive bladder. Arab. J. Urol. 2019, 17, 10-13. [CrossRef] [PubMed]

28. Wibisono, E.; Rahardjo, H.E. Effectiveness of Short Term Percutaneous Tibial Nerve Stimulation for Non-neurogenic Overactive Bladder Syndrome in adults: A Meta-analysis. Acta Med. Indones. 2015, 47, 188-200.

29. Tutolo, M.; Ammirati, E.; van der Aa, F. What is New in Neuromodulation for Overactive Bladder? In European Urology Focus; Elsevier B.V.: Amsterdam, The Netherlands, 2018; Volume 4, pp. 49-53.

30. Lo, C.W.; Wu, M.Y.; Yang, S.S.D.; Jaw, F.S.; Chang, S.J. Comparing the efficacy of onabotulinumtoxinA, sacral neuromodulation, and peripheral tibial nerve stimulation as third line treatment for the management of overactive bladder symptoms in adults: Systematic review and network meta-analysis. Toxins 2020, 12, 128. [CrossRef]

31. Veeratterapillay, R.; Lavin, V.; Thorpe, A.; Harding, C. Posterior tibial nerve stimulation in adults with overactive bladder syndrome: A systematic review of the literature. J. Clin. Urol. 2016, 9, 120-127. [CrossRef]

32. Wang, M.; Jian, Z.; Ma, Y.; Jin, X.; Li, H.; Wang, K. Percutaneous tibial nerve stimulation for overacttive bladder syndrome: A systematic review and meta-analysis. Int. Urogynecol. J. 2020, 31, 2457-2471. 